\title{
Venovenous extracorporeal membrane oxygenation need not be another pain in the neck
}

\author{
Jonathan M. Chen, MD
}

\author{
From the Division of Congenital Cardiac Surgery, Seattle Children's Hospital, Seattle, Wash. \\ Disclosures: Author has nothing to disclose with regard to commercial support. \\ Received for publication March 15, 2018; accepted for publication March 20, 2018; available ahead of print April \\ $21,2018$. \\ Address for reprints: Jonathan M. Chen, MD, Congenital Cardiac Surgery, Seattle Children's Hospital, 4800 Sand \\ Point Way NE, RC2.820, Seattle, WA 98105 (E-mail: jmchen@uw.edu). \\ J Thorac Cardiovasc Surg 2018;156:e15-6 \\ $0022-5223 / \$ 36.00$ \\ Copyright (C) 2018 by The American Association for Thoracic Surgery \\ https://doi.org/10.1016/j.jtcvs.2018.03.057
}

During the past decade, it has become evident that, where feasible, physical therapy-and in particular ambulationduring long periods of extracorporeal membrane oxygenation (ECMO) helps to mitigate progressive deconditioning in otherwise bedbound, critically ill patients. ${ }^{1,2}$ Now considered a fairly routine approach for adult patients on ECMO support (in particular those awaiting lung transplants), this strategy has enjoyed limited adoption in pediatric centers, not only because of technical circuit limitations but in part also because of perceived issues with childhood compliance.

The development of dual-lumen venovenous ECMO cannulas in a variety of sizes has revolutionized cannulation, such that they are now essentially "central lines on steroids." In pediatric patients, however, anatomy can limit appropriate cannula size; exact placement may be delicate to avoid recirculation, migration, or cardiac perforation; and cannula stabilization during periods of mobility is frequently challenging. Although innovative tunneling techniques have been reported, in children most often the right internal jugular vein is used because of superior cannula alignment, leaving patients with a large, unyielding (albeit lifesaving) plastic tube affixed to their necks, not infrequently perilously close to a concomitant tracheostomy site. ${ }^{2}$ In this issue of the Journal, Maeda and colleagues $^{3}$ report a novel cannulation technique for ambulatory long-term ECMO support in 2 pediatric patients (who had failure of dual-lumen venovenous ECMO support) by repurposing Berlin Heart ventricular assist device cannulas (Berlin Heart GmbH, Berlin, Germany) for right atrial and right ventricular access. ${ }^{3}$

The advantages of this new scheme are straightforward - these cannulas were created for long-term use, their implantation is simple yet quite secure, there is virtually no recirculation as long as there is tricuspid competence, and their (arguably more comfortable) exit sites in the upper epigastrium lie far from any potential tracheostomy.

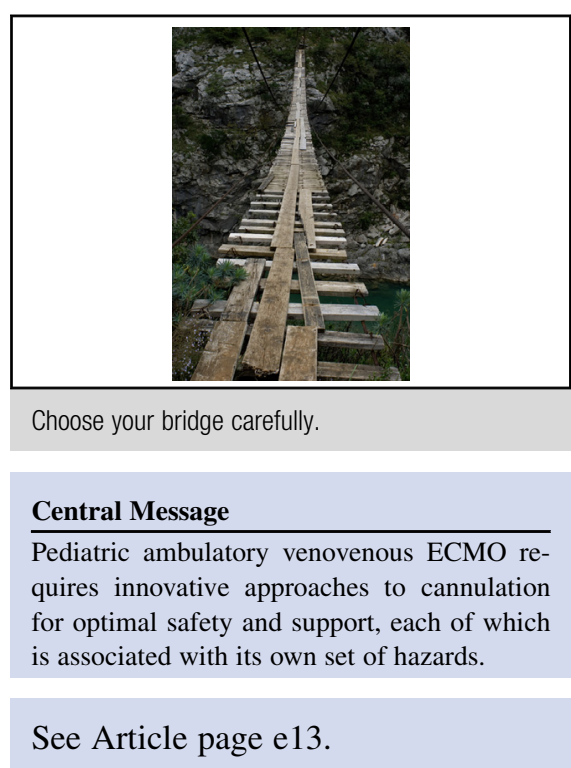

A considerable price is paid, however, because these patients with pulmonary failure required a sternotomy and cardiopulmonary bypass for both implantation and removal 3 to 5 months later. Although neither patient in the series encountered this common complication, any thrombus within the cannulas would surely have mandated a reoperative sternotomy and additional cardiopulmonary bypass, whereas a dual-lumen percutaneous approach would likely have only incurred a Seldinger-type exchange.

As Maeda and colleagues ${ }^{3}$ highlight, their strategy does not off-load (and thus decondition) an otherwise wellfunctioning right ventricle, the integrity of which could be challenged if the ultimate destination is lung transplantation. The converse is also true, however; this strategy (right atrial and right ventricular access) will not ameliorate poor right ventricular function in the manner that early right ventricular ECMO devices had proposed through unloading, and thus it may lack some flexibility because of the location of the outflow limb. ${ }^{4}$

Ambulatory ECMO for children is a logical and laudable goal, no matter where the cannulas emanate, and certainly having a menu of alternative techniques affords surgeons the greatest flexibility. The guiding principle of venovenous ECMO is to bridge the child safely and effectively, whether the destination is recovery or transplant. The choice of the bridge to get there is of utmost importance, however, as each has its own collection of loose planks just waiting to be tested. 


\section{References}

1. Schweickert WD, Pohlman MC, Pohlman AS, Nigos C, Pawlik AJ, Esbrook CL, et al. Early physical and occupational therapy in mechanically ventilated, critically ill patients: a randomised controlled trial. Lancet. 2009;373:1874-82.

2. Garcia JP, Kon ZN, Evans C, Wu Z, Iacono AT, McCormick B, et al. Ambulatory veno-venous extracorporeal membrane oxygenation: innovation and pitfalls. J Thorac Cardiovasc Surg. 2011;142:755-61.
3. Maeda K, Ryan K, Conrad CK, Yarlagadda VV. An alternative cannulation approach for venovenous extracorporeal membrane oxygenation in children for long-term ambulatory support. J Thorac Cardiovasc Surg. 2018;156: e13-4.

4. Wang D, Lick SD, Zhou X, Liu X, Benkowsky RJ, Zwischenberger JB. Ambulatory oxygenator right ventricular assist device for total right heart and respiratory support. Ann Thorac Surg. 2007;84:1699-703. 